\title{
Improvement of speckle correlation fringes by adaptive correction using twisted nematic LCD
}

\author{
Erwin Hack* and Phanindra Narayan Gundu \\ Empa, Laboratory Electronics/Metrology, 129 Überlandstrasse, Duebendorf, CH-8600, Switzerland
}

\begin{abstract}
Subtraction correlation fringes in Digital Speckle Pattern Interferometry are described as a smoothly varying intensity distribution multiplied with high-frequency noise that is due to the random distribution of speckle intensity and speckle phase across the image plane. Although intensity modulation and speckle phase can be eliminated by phase stepping or other phase retrieval methods, noise is not eliminated completely due to the limited dynamic range of image processing, given by the saturation level of the camera, the digitisation depth and the electronic noise. Digital image processing techniques have been developed to reduce the speckle noise from the fringe pattern, but in most cases the fringes are not restored completely. In this paper the speckle noise is reduced before image digitisation by reducing the range of either the modulation intensity values or the speckle phase values, or both, in the interference pattern. This is done adaptively by using programmable twisted nematic LCDs. Since the manipulation is performed for each pixel independently in the reference beam, the imaging quality is retained and no blurring occurs. We quantitatively discuss the improvement and limitation of the method. The experimental verification is performed using an amplitude-only or phase-only spatial light modulator in a conventional ESPI setup.
\end{abstract}

Keywords: DSPI, speckle interferometry, fringe analysis, correlation, LCD, adaptive optics, SLM, noise, SNR

\section{INTRODUCTION}

Electronic or Digital Speckle Pattern Interferometry (ESPI or DSPI) is a widely used non-contact technique for the measurement of displacement, deformation or strain, and non-destructive testing [1]. In DSPI, two interference patterns are recorded digitally with a camera before and after the test object changes its state. The interference is due to the superposition of the speckled wavefront from the object under test and a reference wavefront. Correlation between these two interference patterns by subtraction reveals a fringe pattern, the phase values of which are related to the object change. The correlation fringe pattern is highly noisy owing to the stochastic variations of both phase and amplitude of the speckle field across the image plane. Hence, speckle correlation fringes can be conceived as a smoothly varying intensity signal multiplied to a noise term.

Phase-stepping techniques $[2,3]$ are applied to obtain phase values at each point of the fringe pattern. The deformations can be inferred from the ensuing phase map. But the drawback of these techniques is that they require recording several frames. In some industrial and dynamic applications, a single step correlation method is preferable basically because a controlled laboratory environment may not exist or because the object state may change faster than the quasi-static phase-stepping techniques allow.

The presence of speckle noise limits the practical applications of correlation fringes in DSPI as it is difficult to extract the phase information directly from speckle correlation fringes. The signal-to-noise ratio of a fringe pattern is very low, as shown in section 2 . Therefore, many different digital image processing techniques have been proposed to improve the fringe pattern, including Fourier transform, low-pass and Wiener filtering [4-11]. They induce smoothing of both the noise and the signal. Local averaging and median filtering methods result in image blurring. Recently developed methods based on Wavelet filtering have had some success but the intensity profile of the fringes is not restored completely. Moreover, filtering by Wavelet methods consumes considerable amount of processing time.

\footnotetext{
* erwin.hack@empa.ch; phone+41-44-823 42 73; fax +41-44-823 40 54; www.empa.ch
} 
We developed a technique to reduce the speckle noise before recording of the interference pattern. By pixelwise adaptive adaptation of intensity and/or phase of the reference beam the speckle noise in the correlation fringes is suppressed. Simulations using computer generated speckle correlation fringe patterns show that the combination of adaptive correction to modulation intensity and morphological filtering results in reducing considerably the speckle noise while retaining the fringe shape. The adaptive correction is performed only once before the object deformation is applied. As long as the speckle decorrelation is negligible no update is needed. The main advantages of this adaptive speckle noise reduction are the improvement in the resolution and accuracy of measurement, the faster phase difference acquisition and applicability of DSPI in industrial environment. The first results are obtained with amplitude-only and phase-only spatial light modulators based on a commercial LCD array.

\section{THEORY}

\subsection{Signal to noise ratio in DSPI}

The intensity observed on a CCD pixel where a beam scattered from a rough object interferes with a plane reference beam is given by:

$$
\begin{aligned}
& I_{C C D}=I_{r e f}+I_{s p}+2 \sqrt{I_{r e f} I_{s p}} \cos \left(\varphi_{s p}\right) \\
& \varphi_{s p}=\varphi_{o b j}-\varphi_{r e f}
\end{aligned}
$$

where $I_{r e f}, I_{s p}$, and $\varphi_{s p}$ are the intensity of the reference wavefront, intensity of the speckled wavefront from the object, and the speckle phase, respectively. If we assume that the deformation of the object changes the object phase by an amount $\Delta \varphi$ but leaves the other quantities unchanged, then the modulus of the difference intensity from subtraction correlation is given by

$$
\begin{aligned}
& I(\Delta \varphi)=\left|I_{C C D, \text { final }}-I_{C C D, \text { initial }}\right|=2 I_{M}\left|\sin \left(\varphi_{s p}+\frac{\Delta \varphi}{2}\right)\right|\left|\sin \left(\frac{\Delta \varphi}{2}\right)\right| \\
& I_{M}=2 \sqrt{I_{r e f} I_{s p}}
\end{aligned}
$$

where $I_{M}$ is the modulation intensity. In this speckle fringe pattern the signal, given by the last term in Eq. (2), is multiplied to the stochastic modulation intensity and a stochastic speckle phase term. Therefore, the speckle noise in a DSPI fringe pattern is multiplicative in nature and arises from the presence of two noise terms. Figure 1 illustrates the effect of the different noise terms. The bending of a beam (curve (a)) would give rise to an out-of-plane interference correlation pattern as given by the curve (c). The presence of the multiplicative phase noise term leads to a random signal reduction (curve (b)). If only the modulation intensity noise is considered, we obtain curve (d). Finally, multiplication of both noise terms results in the top curve (e). Note that in this simulation we have some camera saturation, i.e. intensity values greater than 255 grey levels.

To describe the signal to noise ratio (SNR) in a speckle fringe pattern, we compare the expectation value of the intensity for a given phase difference to the variance of the intensity values

$$
S N R_{\text {fringe }}^{2}=\frac{\langle I(\Delta \varphi)\rangle^{2}}{\operatorname{var}[I(\Delta \varphi)]}
$$

where the expectation and variance are taken over all pixels with equal phase difference $\Delta \varphi$. From Eq. (2) we get

$$
S N R_{\text {fringe }}^{2}(\Delta \varphi)=\frac{\left\langle 2 I_{M}\left|\sin \left(\varphi_{s p}+\frac{\Delta \varphi}{2}\right)\right|\right\rangle^{2}}{\operatorname{var}\left[2 I_{M}\left|\sin \left(\varphi_{s p}+\frac{\Delta \varphi}{2}\right)\right|\right]}
$$




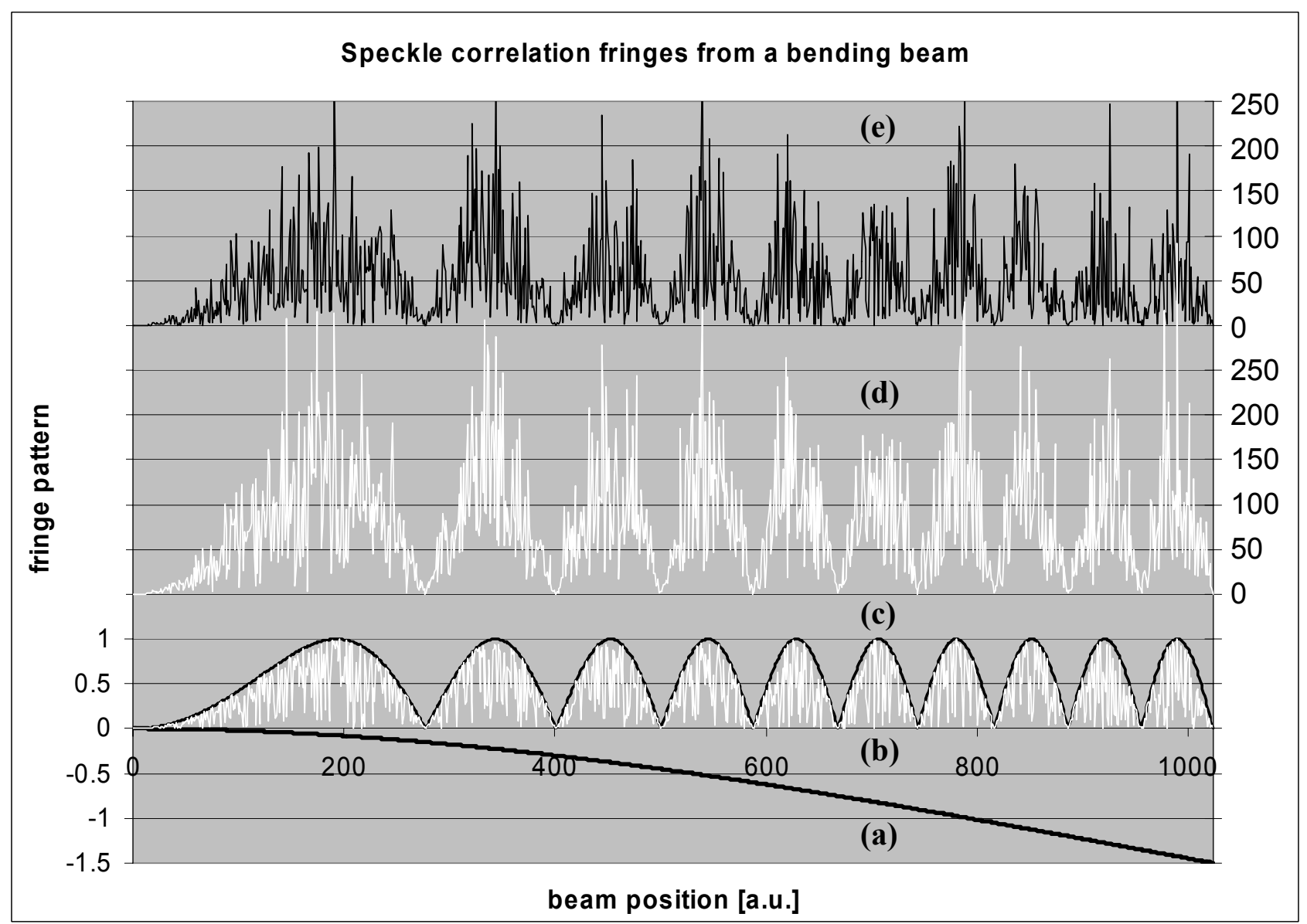

Fig. 1. Cross-section through a simulated fringe pattern. The displacement curve (a), in arbitrary units, for a bending beam, results in the smooth correlation fringe pattern (c). (b) is the fringe pattern with phase noise only, while (d) is the fringe pattern with modulation intensity noise only. (e) is the correlation fringe pattern with both phase noise and modulation intensity noise.

The signal cancels out from Eq. (4) because of the multiplicative nature of the noise. Expectation values and variance are determined by the speckle statistics of the experiment, including surface properties and integration on the camera pixel. After integration, modulation intensity and speckle phase are treated as independent random variables. Assuming the speckle phase to be distributed uniformly among $[0,2 \pi)$, the SNR is independent of the phase difference $\Delta \varphi$, whence

$$
S N R_{\text {fringe }}^{2}=\frac{\left\langle I_{M}\right\rangle^{2}\left\langle\left|\sin \left(\varphi_{s p}\right)\right|\right\rangle^{2}}{\left\langle I_{M}^{2}\right\rangle\left\langle\left|\sin \left(\varphi_{s p}\right)\right|^{2}\right\rangle-\left\langle I_{M}\right\rangle^{2}\left\langle\left|\sin \left(\varphi_{s p}\right)\right|\right\rangle^{2}}
$$

Introducing the individual SNR of the modulation intensity and phase term, defined in analogy to Eq. (3), the SNR of the fringe pattern, Eq.(5), can be expressed by

$$
S N R_{\text {fringe }}^{2}=\frac{S N R_{M}^{2} S N R_{s p}^{2}}{1+S N R_{M}^{2}+S N R_{s p}^{2}}
$$

Eq. (6) shows that the fringe SNR is a monotonous function of the individual SNR of the modulation intensity and the speckle phase term. In other words, increasing one of the SNR will increase fringe SNR as well. If one of the individual SNR is dominant, i.e. $S N R_{M}^{2} \ll S N R_{s p}^{2}$ or $S N R_{M}^{2} \gg S N R_{s p}^{2}$ the other SNR becomes the limiting SNR of the fringe. 
The probability density function of the modulation intensity when a smooth reference wave is used is given by [12]

$$
p\left(I_{M}\right)=\frac{n I_{M}}{2 I_{r e f}\left\langle I_{s p}\right\rangle} \exp \left(-\frac{n I_{M}^{2}}{4 I_{r e f}\left\langle I_{s p}\right\rangle}\right) \text { for } n \geq 1
$$

where $n$ is the average number of speckles per pixel. The first moments are

$$
\left\langle I_{M}\right\rangle^{2}=\frac{\pi}{n} I_{r e f}\left\langle I_{s p}\right\rangle \text { and }\left\langle I_{M}{ }^{2}\right\rangle=\frac{4}{n} I_{r e f}\left\langle I_{s p}\right\rangle
$$

Inserting Eq. (8) into the expression for the SNR of the modulation intensity, it is seen that

$$
S N R_{M}^{2}=\frac{\left\langle I_{M}\right\rangle^{2}}{\left\langle I_{M}^{2}\right\rangle-\left\langle I_{M}\right\rangle^{2}}=\frac{\pi}{4-\pi}=3.66
$$

is independent of the number of speckles per pixel. To calculate the SNR of the speckle phase term, the distribution of the speckle phase values is assumed to be rectangular, so that the distribution of the sine-values gives

$$
\left\langle\left|\sin \left(\varphi_{s p}\right)\right|\right\rangle^{2}=0.348 \text { and }\left\langle\left|\sin \left(\varphi_{s p}\right)\right|^{2}\right\rangle=0.454
$$

resulting in a SNR for the speckle phase term of $S N R_{s p}^{2}=3.31$. This value is comparable to the SNR of the modulation intensity. Note that with these values inserted into Eq. (6) the SNR for a general DSPI fringe then is only $S N R_{\text {fringe }}^{2}=1.52$.

\section{EXPERIMENTAL}

\subsection{Speckle noise reduction before image capture}

An improvement in the SNR of the fringe pattern can be obtained by limiting either the variance (range of values) of the modulation intensity $I_{M}$ or of the random speckle phase $\varphi_{s p}$, or both. When using liquid crystal arrays whose pixels are matched to the CCD-camera [13], phase and amplitude of the transmitted wave can be independently controlled pixel by pixel. An experimental set-up is illustrated in Fig.2. The twisted nematic LCD (TN-LCD) is placed in the reference beam. Its pixels are imaged onto the CCD camera one to one, as illustrated by the rays, together with the corresponding area on the test object. Hence, the reference beam intensity and phase can be adjusted pixelwise in order to realize the adaptive correction of the correlation fringes.

On one hand, $I_{M}$ can be calculated according to Eq. (2) from measuring $I_{r e f}$ with transparent LCD and $I_{s p}$ separately, with beam shutters. Knowing the individual value of $I_{M}$ in each pixel of the fringe pattern, it can be modified by varying $I_{\text {ref }}$ according to Eq. (2). In the ideal case, constant modulation intensity can be obtained, i.e. $\operatorname{var}\left[I_{M}\right]=0$. Apart from an improvement in the SNR, constant modulation intensity transforms the speckle correlation fringe pattern into a well defined envelope, as illustrated in Fig. 1, graph (c). The remaining noise (speckle phase noise) can be further reduced by applying morphological filtering [14].

On the other hand, the speckle phase can be measured in each pixel before the experiment. This can be accomplished by using the phase-only LCD as a phase-stepping device. Then the phase-only LCD is programmed such that the speckle phase is compensated, resulting ideally in $\operatorname{var}\left[\varphi_{s p}\right]=0$. In this case, the fringe pattern is

$$
I(\Delta \varphi)=2 I_{M} \sin ^{2}\left(\frac{\Delta \varphi}{2}\right)
$$




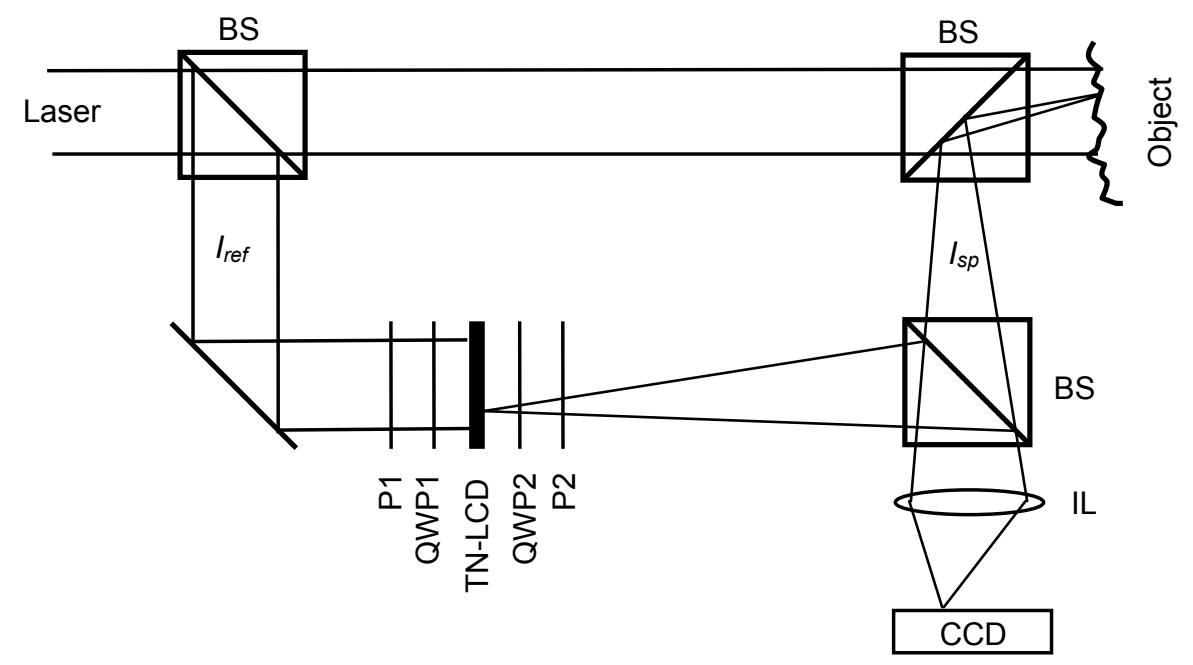

Fig. 2. Experimental realization of adaptive speckle correction. BS are non-polarizing beam splitters, P1 and P2 are linear polarizers, QWP1 and QWP2 are quarter-waveplates, TN-LCD is the twisted-nematic LCD, and IL is the imaging lens.

as illustrated in Fig. 1, graph (d).

\subsection{Liquid crystal device as spatial light modulator}

Commercial LCDs are intended only for varying intensity, but they also change the phase values of the transmitted light in an undefined manner. Since we require amplitude-only or phase-only modulation, the LCD is sandwiched between two quarter-wave plates (QWP1, QWP2) and polarizers (P1, P2) to produce elliptically polarized light [16, 17], as illustrated in Fig.2. We use a SVGA resolution twisted nematic LCD from Sony (LCX016AL) having 832 x 624 pixels with pixel pitch of $32 \mu \mathrm{m}$ and 1.3 inch diagonal size. This LCD setup is inserted in a Mach-Zehnder interferometer as shown in Fig. 2. The addressing of the LCD is done electrically using the graphic board of the PC. The orientation of the quarter-wave plates and polarizers are optimized to obtain an amplitude-only or phase-only SLM, depending on the intended use.

The maximum intensity modulation obtained with our LCD in amplitude-only mode is $96 \%$, i.e. the transmission factor is scalable from 0.04 to 1.0. How to find the optimum modulation intensity in this non-ideal situation has been reported elsewhere [15].

The maximum phase change obtained with our LCD in phase-only mode is more than $\pi \mathrm{rad}$. This is sufficient to correct the speckle phases, since from Eq. (2) we need only the modulus of the sine-function for which $\left|\sin \left(\varphi_{s p}+\frac{1}{2} \Delta \varphi\right)\right|=\left|\sin \left(\varphi_{s p}+\frac{1}{2} \Delta \varphi+\pi\right)\right|$. In order to measure the speckle phase, the phase-only LCD itself is used as the phase shifter. A three frame phase stepping method is chosen with phase step values of $0, \pi / 2$ and $\pi[16]$.

\section{CONCLUSION}

Theoretical and experimental investigations have shown the feasibility of improving speckle fringe patterns by pixelwise modulation of the $I_{M}$ values or speckle phase compensation with an amplitude-only or phase-only LCD SLM, respectively. The SNR can be improved significantly. The technique is limited by the dynamic range of the LCD. Working with LCDs and CCDs with higher dynamic range and higher bit quantization will be useful. Further improvements of the fringe SNR are expected by using two LCD SLMs in a 4F processor. 


\section{REFERENCES}

1. P.K. Rastogi (Ed.), Digital speckle pattern interferometry and related techniques, John Wiley \& Sons Ltd, Chichester, 2001.

2. K. Creath, "Phase-shifting speckle interferometry", Appl. Opt. 24, 3053-3058 (1985).

3. Y. Surrel, "Design of algorithms for phase measurements by the use of phase stepping", Applied Optics 35, 51-60 (1996).

4. H.H. Arsenault and G. April, "Speckle removal by optical and digital processing", Journal of Optical Society of America 66, 177 (1976).

5. A.K. Jain, C.R. Christensen, "Digital processing of images in speckle noise", Proc. SPIE 243, $46-50$ (1980).

6. J.S. Lim, H. Nawab, "Techniques for speckle noise removal”, Proc. SPIE 243, 35-44 (1980).

7. P. Varman, C. Wykes, "Smoothing of speckle and moiré fringes by computer processing", Opt. Las.Eng. 3, 87-100 (1981).

8. D. Kerr, F.M. Santoyo, J.R. Tyrer, "Manipulation of Fourier components of speckle fringe patterns as part of an interferometric analysis process", J. Modern Optics 36, 195-203 (1989).

9. K. Lebart and J.M. Boucher, "Speckle filtering by wavelet analysis and synthesis", Proc. SPIE 2825, 644-651 (1996).

10. A. Federico, G.H. Kaufmann, "Comparative study of wavelet thresholding methods for denoising electronic speckle pattern Interferometry fringes", Opt. Eng. 40, 2598-2604 (2001).

11. R. Kumar, I.P. Singh, and C. Shakher, "Measurement of out-of-plane static and dynamic deformations by processing digital speckle pattern interferometry fringes using wavelet transform”, Opt. Las. Eng. 41, 81-93 (2004).

12. M. Lehmann, "Phase-shifting speckle interferometry with unresolved speckles: A theoretical investigation", Optics Communication 128, 325-340 (1996)

13. G. Wernicke, S. Krüger, H. Gruber, N. Demoli, M. Dürr, and S. Teiwes, “ Liquid crystal display as spatial light modulator for diffractive optical elements and the reconstruction of digital holograms", Proc. SPIE 4596, 182-190 (2001)

14. E. Hack, P.N. Gundu, and P.K. Rastogi, "Adaptive correction to the speckle correlation fringes using twisted nematic LCD“, Appl. Opt. 44, 2772-2781 (2005)

15. E. Hack and P.N. Gundu, "Adaptive correction to the speckle correlation fringes using twisted nematic LCD, Proc. FRINGE 2005, Ed. W. Osten, Springer, Berlin, 2005, 158-165

16. P.N. Gundu, E. Hack, P.K. Rastogi, "Adaptive technique for phase noise reduction from the speckle correlation fringes", in: Optical Information Systems III; B. Javidi and D. Psaltis; Eds., Proc. SPIE 5908, 263-269 (2005)

17. J.L. Pezzaniti and R.A. Chipaman, "Phase-only modulation of a twisted nematic liquid-crystal TV by use of the eigenpolarization states", Opt. Lett. 18, 1567-1569 (1993).

18. I. Moreno, J.A. Davis, K.G. D'Nelly, and D.B. Allison, "Transmission and phase measurement for polarization eigenvectors in twisted-nematic liquid crystal spatial light modulators", Opt. Eng. 37, 3048-3052 (1998) 